\title{
Covid-19: impacto na saúde mental da população em tempos de pandemia, uma revisão integrativa
}

\author{
Covid-19: impact on the mental health of the population in times of pandemic, an integrative \\ review
}

Covid-19: impacto en la salud mental de la población en tiempos de pandemia, una revisión integradora

Recebido: 27/11/2021 | Revisado: 03/12/2021 | Aceito: 03/12/2021 | Publicado: 04/12/2021

\author{
Ligia dos Santos Brasil \\ ORCID: https://orcid.org/0000-0003-3063-092X \\ Centro Universitário da Amazônia, Brasil \\ E-mail: ligiabrasil19@gmail.com \\ Márcia Eline Rayol \\ ORCID: https://orcid.org/0000-0001-9388-7066 \\ Centro Universitário da Amazônia, Brasil \\ E-mail: marciaelinerayol2@gmail.com \\ Maria da Conceição Caetano Siqueira \\ ORCID: https://orcid.org/0000-0003-1731-4076 \\ Centro Universitário da Amazônia, Brasil \\ E-mail: siqueira_44@hotmail.com
}

\begin{abstract}
Resumo
A pandemia por Covid-19 apresenta-se além da sintomatologia respiratória, os sintomas de transtorno de estresse póstraumático (TEPT), ansiedade e depressão também vêm sendo prevalentes em pacientes infectados. estudo de revisão integrativa da literatura, tendo como foco a pesquisa bibliográfica descritiva, retrospectiva e qualitativa a partir de estudo, análise e correlação de material já publicado para embasamento ao tema proposto e busca investigar o impacto da pandemia a saúde mental da população. Os principais destaques foram para: ansiedade, depressão, uso de drogas, propensão ao suicídio. Foi possível observar ainda, que as ocorrências de ordem emocionais por vezes não são advindas diretamente da doença em si, dos sintomas e sinais, mas estão fortemente associadas a todas as desordens biológicas, psicológicas e sociais que a pandemia acabou trazendo nos últimos dois anos. foi possível observar o aumento de queixas como: ansiedade, depressão, estresse pós traumático. Além das já citadas, ainda foi observada o aumento do abuso de drogas, assim como a propensão ao suicídio, e o ato em si. Diante disso, se faz necessário que o sistema de saúde construa políticas públicas que venham a atender a população na saúde mental pós Covid-19, além da divulgação de informações corretas sobre o tratamento e a prevenção à patologia, uma vez que a melhor forma de prevenção é a educação em saúde.
\end{abstract}

Palavras-chave: Covid-19; Assistência à saúde mental; Pandemia.

\begin{abstract}
The Covid-19 pandemic presents, in addition to respiratory symptoms, symptoms of post-traumatic disorder (PTSD), anxiety and depression that are also strong, being prevalent in infected patients. integrative literature review study, focusing on descriptive, retrospective and qualitative bibliographic research based on the study, analysis and correlation of material already published to support the proposed theme and seeks to investigate the impact of the pandemic on the mental health of the population. The main highlights were: anxiety, depression, drug use, propensity to commit suicide. It was also possible to observe that emotional occurrences sometimes do not arise directly from the disease itself, symptoms and signs, but are strongly associated with all the biological, psychological and social disorders that a pandemic brought about in the last two years. it was possible to observe an increase in complaints such as: anxiety, depression, post traumatic stress. In addition to those already mentioned, an increase in drug abuse was also observed, as well as the propensity to commit suicide, and the act itself. Therefore, it is necessary for the health system to build public policies that come to serve the population in mental health after Covid-19, in addition to the dissemination of correct information about the treatment and prevention of the pathology, as the best form of prevention is health education.
\end{abstract}

Keywords: Covid-19; Mental health care; Pandemic. 


\begin{abstract}
Resumen
La pandemia de Covid-19 presenta, además de síntomas respiratorios, síntomas de trastorno postraumático (TEPT), ansiedad y depresión que también son fuertes, siendo prevalentes en pacientes infectados. Estudio de revisión integradora de la literatura, con foco en la investigación bibliográfica descriptiva, retrospectiva y cualitativa basada en el estudio, análisis y correlación de material ya publicado para sustentar la temática propuesta y busca investigar el impacto de la pandemia en la salud mental de la población. Los principales destaques fueron: ansiedad, depresión, consumo de drogas, propensión al suicidio. También se pudo observar que las ocurrencias emocionales en ocasiones no surgen directamente de la propia enfermedad, síntomas y signos, sino que están fuertemente asociadas a todos los desórdenes biológicos, psicológicos y sociales que una pandemia provocó en los últimos dos años. se pudo observar un aumento de quejas como: ansiedad, depresión, estrés postraumático. Además de los ya mencionados, también se observó un aumento del uso indebido de drogas, así como la propensión al suicidio, y el acto en sí. Por ello, es necesario que el sistema de salud construya políticas públicas que vengan a servir a la población en salud mental posterior al Covid-19, además de la difusión de información correcta sobre el tratamiento y prevención de la patología, como la mejor forma de prevención. es educación para la salud.
\end{abstract}

Palabras clave: Covid-19; Cuidado de la salud mental; Pandemia.

\title{
1. Introdução
}

Recentemente o mundo inteiro ficou estarrecido diante da descoberta de um novo Coronavírus, para o qual a humanidade não possui imunidade e nem conhecimentos sobre tratamento especifico, e por ter alta taxas de transmissão, os serviços de saúde de diferentes países entraram em descensão. Desde que foi detectado o primeiro caso no final de 2019 , o vírus tem se espalhado rapidamente no mundo, no dia 30 de janeiro de 2020, a Organização Mundial de Saúde (OMS) classificou a doença provocada pelo vírus, Covid-19, como uma emergência de saúde internacional, no dia 11 de março, a OMS declarou haver uma pandemia de Covid-19. (Hallal et al. 2020).

Os acontecimentos dos últimos dois anos vêm acometendo bruscamente a saúde mental das pessoas de todas as idades e classes sociais, o avanço da doença vem contribuído para o acréscimo dos distúrbios mentais, sendo relevante dar visibilidade ao adoecimento mental população. É autentico que a pandemia de Covid-19 provocou desordem, acarretou impacto em nível global, cenário de guerra nos hospitais, sobrecarga os profissionais da saúde, perdas afetivas e materiais imensuráveis, isolamento social e a incertezas quanto ao futuro, muitos perderam seus empregos, escolas, faculdades, bares, restaurantes, boates e locais de culto não puderam mas ser frequentados, e qualquer atividade que promova aglomerações estão sendo evitadas para conter a transição, tais medidas suscitaram substancial impacto na sociedade, posto que havia uma rotina no dia a dia, trabalhos, estudos, lazeres, desta forma o isolamento se tornou um desafio (Brooks et al. 2020)

A pandemia por Covid 19 apresenta-se além da sintomatologia respiratória, os sintomas de transtorno de estresse póstraumático (TEPT), ansiedade e depressão também vêm sendo prevalentes em pacientes infectados com Covid-19. Diante de tais dados, faz se necessário peguilhar as evidências sobre os impactos negativos da pandemia sobre a saúde mental da população, dado que milhões de pessoas em todo o mundo são isoladas em quarentena para minimizar a transmissão do SARSCoV-2.

Raony et al. (2020), afirma que é presumível que tanto a infecção por SARS-CoV-2 quanto as medidas epidemiológicas e todos os demais transtornos causados pela pandemia possam levar a mudanças nos circuitos psiconeuroendócrinos-imunes com impacto no aparecimento e / ou evolução de deficiências de saúde mental em indivíduos infectados bem como naqueles indivíduos que, apesar de não estejam infectados, estão sujeitos ao isolamento social. Em face do exposto, questiona-se: "qual o impacto da "covid 19 na saúde mental da população?".

Diante disso, este estudo tem por objetivo analisar o impacto na saúde mental da população em função da Covid-19 identificando as alterações psíquicos na população, afim de descrever os impactos obtidos pela doença através da análise dos resultados encontrados. 


\section{Metodologia}

Trata-se de um estudo de revisão integrativa da literatura, tendo como foco a pesquisa bibliográfica descritiva, retrospectiva e qualitativa a partir de estudo, análise e correlação de material já publicado para embasamento ao tema proposto e busca investigar o impacto da pandemia a saúde mental da população.

De acordo com Souza et al. (2010), a revisão integrativa é um método que constitui basicamente um instrumento da prática baseada em evidências é a mais ampla abordagem metodológica no que se referente às revisões de literatura, isto permite a inclusão de estudos experimentais e não-experimentais para uma compreensão maior e mais completa do fenômeno analisado.

No que se refere ao aspecto descritivo, Gerhardt e Silveira (2009), consideram que o estudo descritivo é uma das classificações das pesquisas científicas, com o objetivo de descrever as características do objeto de estudo, um fenômeno ou experiência para o estudo completo.

Para coleta de dados foi realizado um levantamento de informações relacionados ao objeto de estudo, através de artigos em bases de dados eletrônicos: BVS (Biblioteca Virtual em Saúde), SciELO (Scientific Electronic Library Online) e Lilacs (Literatura latino-americana e do caribe em ciências da saúde), com uso do descritor "covid 19”, associado ao outro descritor, "saúde mental" suas respectivas traduções para o inglês.

Os critérios de inclusão utilizados se darão por produções completas na modalidade de artigos científicos, teses de doutorados, dissertações de mestrados e materiais do Ministério da Saúde disponíveis na internet e gratuitos, nas línguas portuguesa e inglesa que contemplem o tema proposto, no período de 2018 a 2021.

Os critérios de exclusão foram: As publicações que não se encontravam disponíveis em texto completo; as publicações que apresentavam disponibilidade de texto completo, mas cujo o link apresentava erro mediante a tentativa de acessá-lo e os artigos fora do período determinado 2018 a 2021.

A busca na plataforma Scielo com o descritor Covid-19 associado ao descritor saúde mental, foram obtidos 16 artigos, na base de dados Lilacs 106 literaturas, na BVS a pesquisa resultou em 432. Ao aplicar o primeiro critério de inclusão, publicações entre os de 2018 a 2021, a plataforma Scielo, Lilacs e BVS mantiveram o mesmo número de publicações, após segundo critério, publicações nas línguas portuguesa e inglesa, na Scielo se obteve 7 artigos, na Lilacs 94 resultado, na BVS 413, após o terceiro critério, produções completas na modalidade de artigos científicos, Scielo se manteve na mesma quantidade de artigos, Lilacs 93 e BVS 402 publicações, após ser aplicado o quarto critério , analise dos títulos dos artigos que contemplem o tema proposto, na plataforma Scielo se obteve 3 artigos, Lilacs 18, BVS 120 artigos. Teve como resultado a análise dos resumos simples, Scielo 1 e Lilacs 6 e BVS se obteve 6 artigos.

A coleta de dados dos artigos se deu entre os meses de setembro a outubro de 2021 e foi utilizada uma listagem dos artigos encontrados com título do artigo, local de publicação, objetivos, autores e ano de publicação.

A amostra final desta revisão integrativa foi constituída por 554 artigos onde foram selecionados 13 artigos para exposição.

A análise de conteúdo levou em consideração o foi proposto por Bardin (2012), no que se refere análise de conteúdo, a obra de Bardin se constitui uma importante ferramenta e através dela é possível se obter um melhor resultado após aplicação do mesmo. O método se constitui de um conjunto de técnicas de análise de comunicações, que utiliza alguns processos sistemáticos e objetivos de descrição do conteúdo das mensagens. Em sua obra, Azevedo et al. (2021), sintetiza as fases de análise de dados (Figura 1). 
Figura 1. Compilado das fases do método de análise de conteúdo de Bardin.

\begin{tabular}{c|l}
\hline \multicolumn{2}{c}{ MÉTODO DE ANÁLISE DE CONTEÚDO } \\
\hline $\begin{array}{c}\text { FASE I } \\
\text { Pré - análise }\end{array}$ & $\begin{array}{l}\text { Seleção dos materiais que tem relação com o objeto de estudo, embasamento argumentativos } \\
\text { para os resultados obtidos. Nesta fase são aplicados os critérios de inclusão e exclusão, para } \\
\text { facilitar a obtenção de dados que serão realmente aproveitados. }\end{array}$ \\
\hline $\begin{array}{c}\text { FASE II } \\
\text { maração do } \\
\text { material }\end{array}$ & $\begin{array}{l}\text { Estudo e leitura minuciosa dos materiais obtidos. Nesta fase são avaliação os escritos e } \\
\text { selecionados os que serão necessários para sustentar do tema de estudo, assim como os que } \\
\text { serão utilizados para fazer correlação com a proposta do tema em questão. }\end{array}$ \\
\hline $\begin{array}{c}\text { FASE III } \\
\text { Tratamento dos } \\
\text { resultados }\end{array}$ & $\begin{array}{l}\text { Validação dos dados analisados. Relação dos objetivos com os resultados, e possivel } \\
\text { surgimento de novas hipóteses. }\end{array}$ \\
\hline
\end{tabular}

Fonte: Azevedo et al. (2021).

\section{Resultados e Discussões}

Na presente revisão integrativa foram analisados 13 artigos que estavam de acordo com os critérios de inclusão préestabelecidos. A classificação das referências encontradas (Tabela 1) foi feita segundo o código do artigo, autores/ano, local de publicação e objetivo de cada artigo.

Tabela 1. Artigos selecionados para o estudo.

\begin{tabular}{|c|c|c|c|c|}
\hline $\mathbf{N}$ & $\begin{array}{l}\text { AUTOR/ } \\
\text { ANO }\end{array}$ & $\begin{array}{c}\text { LOCAL DA } \\
\text { PUBLICAÇÃ̃O }\end{array}$ & TÍTULO & OBJETIVO DO ESTUDO \\
\hline $\mathrm{C} 1$ & $\begin{array}{l}\text { Pavani et al. } \\
\quad(2020)\end{array}$ & $\begin{array}{l}\text { Revista gaúcha de } \\
\text { enfermagem }\end{array}$ & $\begin{array}{c}\text { Covid-19 e as repercussões } \\
\text { na saúde mental: estudo de } \\
\text { revisão narrativa de } \\
\text { literatura. }\end{array}$ & $\begin{array}{l}\text { Identificar as repercussões na saúde mental de } \\
\text { grupos e populações no contexto da pandemia do } \\
\text { novo coronavírus. }\end{array}$ \\
\hline $\mathrm{C} 2$ & $\begin{array}{l}\text { Alvarez \& } \\
\text { Toro. }(2021)\end{array}$ & $\begin{array}{l}\text { Repertório de } \\
\text { medicina e cirurgia }\end{array}$ & $\begin{array}{l}\text { Impacto do covid-19 na } \\
\text { saúde } \\
\text { Mental: revisão da literatura } \\
\end{array}$ & $\begin{array}{l}\text { Descrever o impacto o covid-19 na saúde mental a } \\
\text { partir da revisão da literatura científica. }\end{array}$ \\
\hline $\mathrm{C} 3$ & $\begin{array}{l}\text { Santos e } \\
\text { Rodrigues. } \\
\text { (2020) }\end{array}$ & Revista nursing & $\begin{array}{l}\text { Covid-19 e repercussões } \\
\text { psicológica durante a } \\
\text { quarentena e o isolamento } \\
\text { social: uma revisão } \\
\text { integrativa }\end{array}$ & $\begin{array}{l}\text { Analisar os principais impactos psicológicos } \\
\text { decorrente da quarentena cedo isolamento social }\end{array}$ \\
\hline $\mathrm{C} 4$ & $\begin{array}{l}\text { Moni et al. } \\
\quad(2021)\end{array}$ & Plos one & $\begin{array}{l}\text { Sofrimento psicológico, } \\
\text { medo e enfrentamento entre } \\
\text { Malaios durante a pandemia } \\
\text { Covid-19 }\end{array}$ & $\begin{array}{l}\text { Investigar o sofrimento psíquico, o medo e as } \\
\text { estratégias de enfrentamento em decorrência da } \\
\text { pandemia Covid- } 19 \text {. }\end{array}$ \\
\hline C5 & $\begin{array}{l}\text { Richter et al. } \\
\qquad(2021)\end{array}$ & $\begin{array}{l}\text { Epidemiology and } \\
\text { psychiatric sciences }\end{array}$ & $\begin{array}{l}\text { Problemas de saúde mental } \\
\text { em geral população durante } \\
\text { e após o primeiro bloqueio } \\
\text { fase devido à pandemia sars- } \\
\text { cov-2: rápida } \\
\text { Revisão de estudos de } \\
\text { múltiplas ondas } \\
\end{array}$ & $\begin{array}{c}\text { Revisar estudos sobre o curso de problemas de } \\
\text { saúde mental durante e após o } \\
\text { Primeira fase de bloqueio }\end{array}$ \\
\hline C6 & $\begin{array}{l}\text { Filho e Lima } \\
\qquad(2020)\end{array}$ & $\begin{array}{c}\text { Gerência de } \\
\text { Informações } \\
\text { Estratégicas em } \\
\text { Saúde CONECTA- } \\
\text { SUS } \\
\end{array}$ & $\begin{array}{l}\text { Saúde mental na pandemia } \\
\text { covid-19. }\end{array}$ & $\begin{array}{l}\text { Avaliar os efeitos psicológicos na população geral } \\
\text { durante os estágios iniciais da epidemia }\end{array}$ \\
\hline $\mathrm{C} 7$ & $\begin{array}{l}\text { Pinheiro e } \\
\text { Kocourek } \\
(2020)\end{array}$ & Revista Cuidarte & $\begin{array}{l}\text { Saúde mental em tempos de } \\
\text { pandemia: qual o impacto do } \\
\text { Covid-19? }\end{array}$ & $\begin{array}{l}\text { Analisar a saúde mental da população em tempos } \\
\text { de pandemia. }\end{array}$ \\
\hline $\mathrm{C} 8$ & Rodríguez & Revista cientifica & Impacto do Covid-19 na & Descrever de uma forma geral o impacto do Covid- \\
\hline
\end{tabular}




\begin{tabular}{|c|c|c|c|c|}
\hline & $(2020)$ & vila clara. & saúde mental das pessoas & 19 na saúde mental das pessoas. \\
\hline C9 & $\begin{array}{l}\text { Nochaiwong } \\
\text { et al. (2021) }\end{array}$ & $\begin{array}{l}\text { Relatórios } \\
\text { Científicos }\end{array}$ & $\begin{array}{c}\text { Prevalência global de } \\
\text { doenças mentais problemas } \\
\text { de saúde entre a geral } \\
\text { população durante o } \\
\text { coronavírus doença- } \\
\text { pandemia de 2019: uma } \\
\text { revisão sistemática e meta- } \\
\text { análise } \\
\end{array}$ & $\begin{array}{l}\text { Analisar a prevalência global contemporânea de } \\
\text { problemas de saúde mental entre a população em } \\
\text { geral em meio à pandemia de doença coronavírus- } \\
2019 \text { (Covid-19). }\end{array}$ \\
\hline $\mathrm{C} 10$ & $\begin{array}{l}\text { Galea et al. } \\
\quad(2021)\end{array}$ & $\begin{array}{l}\text { Publishing a } \\
\text { Supplement - } \\
\text { American Journal of } \\
\text { Public Health }\end{array}$ & $\begin{array}{l}\text { Saúde Mental e Mortalidade } \\
\text { em um Tempo de Covid-19 }\end{array}$ & $\begin{array}{l}\text { Analisar o impacto na saúde mental relacionado } \\
\text { com a alta mortalidade por Covid } 19 .\end{array}$ \\
\hline $\mathrm{C} 11$ & $\begin{array}{l}\text { Ouyang et al. } \\
\text { (2021) }\end{array}$ & Science Progress & $\begin{array}{l}\text { Avaliação do efeito da } \\
\text { Pandemia de Covid-19 em } \\
\text { características clínicas e } \\
\text { estado psicológico em } \\
\text { consulta de internet } \\
\text { respondentes }\end{array}$ & $\begin{array}{l}\text { Investigar as características clínicas e psicológicas } \\
\text { situação de uma população específica usando uma } \\
\text { plataforma de consulta na Internet durante o surto } \\
\text { de Covid-19 }\end{array}$ \\
\hline $\mathrm{C} 12$ & $\begin{array}{l}\text { Prado e } \\
\text { Freitas } \\
(2020)\end{array}$ & $\begin{array}{l}\text { Estudos de } \\
\text { Psicologia }\end{array}$ & $\begin{array}{c}\text { Distanciamento social } \\
\text { durante pandemias: risco de } \\
\text { suicídio e prevenção em face } \\
\text { dos impactos psicossociais } \\
\text { da Covid-19 }\end{array}$ & $\begin{array}{l}\text { Apresentar as implicações negativas da pandemia } \\
\text { de Covid-19 para a saúde mental e articulá-las ao } \\
\text { risco de suicídio na população geral. }\end{array}$ \\
\hline $\mathrm{C} 13$ & $\begin{array}{l}\text { Varma et al. } \\
\text { (2021) }\end{array}$ & $\begin{array}{l}\text { International Journal } \\
\text { of Environmental } \\
\text { Research and Public } \\
\text { Health }\end{array}$ & $\begin{array}{l}\text { Má qualidade do sono e sua } \\
\text { relação com características } \\
\text { individuais, experiências } \\
\text { pessoais e saúde mental } \\
\text { durante a pandemia de } \\
\text { Covid-19 }\end{array}$ & $\begin{array}{c}\text { Determinar as diferenças de linguagem usadas para } \\
\text { descrever experiências pessoais de bons dormidores } \\
\text { em comparação com maus dormidores, e explorar } \\
\text { associações entre saúde mental e sono insatisfatório. } \\
\text { Características demográficas específicas e fatores } \\
\text { relacionados à pandemia, como solidão, mudanças } \\
\text { negativas na situação financeira e o risco de contrair } \\
\text { a doença Covid-19 também foram examinados como } \\
\text { covariáveis de associações entre saúde mental e sono } \\
\text { insatisfatório. }\end{array}$ \\
\hline
\end{tabular}

Fonte: Brasil, Rayol e Siqueira (2021).

Após a análise dos 13 artigos, emergiram dois eixos temáticos capazes de sinalar as evidencias cientificas disponíveis nas literaturas sobre a temática abordada, conforme pode ser visto a seguir:

\subsection{Alterações psíquicas encontradas.}

\subsubsection{Ansiedade}

Pavani et al. (2020), em sua pesquisa bibliográfica, afirma que a pandemia contribuiu para o agravamento da ansiedade na população, segundo seu trabalho os públicos, mas afetados são, os estudantes e trabalhadores imigrantes, mulheres, idosos, pessoas com problemas psiquiátricos anteriores e / ou pessoas institucionalizadas, e os de 18 a 30 anos.

Alvarez e Jorge (2021), destacam em sua pesquisa alguns afetos negativos observados na população em geral, dentre eles sintomas manifestos de ansiedade, propensão ansiedade, transtorno de ansiedade generalizada, ansiedade fóbica.

Santos (2020), em seu estudo demonstrou que o isolamento social implicou em mudanças na rotina, ou seja, empecilho na realização de atividades corriqueiras e que repercutem negativamente na saúde mental, levando a agravos como a ansiedade.

Moni et al. (2021), associou a dificuldade financeira por conta da perda de empregos, em virtude das medidas de contenção do vírus à sintomas da ansiedade.

Richter et al. (2021), corroboram que em termos de psicopatologia, encontrou-se em sua pesquisa várias doenças mentais, uma das mais comum abordas foi a ansiedade. 
Filho e Lima (2020), avaliaram os efeitos psicológicos na população geral durante os estágios iniciais da epidemia, verificaram taxas elevadas de ansiedade.

Pinheiro e Kocourek (2020), observaram em sua pesquisa que pessoas que evoluem para um quadro infeccioso grave, ficam distanciadas da família por estarem internadas ou isoladas em casa. Em casos que evolução para o óbito a família fica impedida de fazer os ritos de despedida, gerando sofrimento e dificuldade em lidar o luto, desencadeando desta forma transtornos psicossomáticos, principalmente a ansiedade.

Rodríguez (2020), afirma que as saúdes mentais da população em geral podem ser significativamente afetadas pela pandemia do Covid-19, como resultado de níveis mais elevados de estresse e ratifica que a Covid-19 pode afetar toda a sociedade e que qualquer pessoa que deve sua vida de alguma forma afetada pelos impactos da pandemia pode ter consequências psicossociais, principalmente os grupos mais vulneráveis como os adolescentes.

Em sua análise Nochaiwong et al. (2021), destacou o aumento da ansiedade, tanto entre paciente, quanto em familiares, demonstrando que a patologia não afeta mentalmente apenas o paciente, mas todo o núcleo familiar. Em sua obra, Galea e Ettman (2021), corroboram que com a pandemia ocorreu um aumento 3 vezes maior de transtornos de ansiedade e humor.

A obra de Ouyang et al. (2021), apontou que a incidência de a ansiedade foi de 54,15\%, este resultado é maior do que a da população em geral. O mesmo autor afirma ainda que o fechamento dos postos de trabalho devido as restrições de circulação e distanciamento social, contribuíram diretamente para o resultado obtido. A incidência de ansiedade na população permanece elevada por conta do estresse da pandemia, dessa forma se faz necessário adotar intervenções psicológicas precisas e medidas de educação em saúde.

\subsubsection{Depressão}

Pavani et al. (2020) observou o aumento da depressão e o agravamento da população com problemas anteriores. A depressão foi evidenciada quanto em pessoas com diagnóstico positivo para a covid como também aquelas que tinham parentes ou amigos infectados ou que obtiveram algum tipo de prejuízo com a pandemia.

Alvarez e Jorge (2021), em seu trabalho de pesquisa pode observar diversos afetos que o mesmo considera negativo para a saúde mental, entre eles transtorno depressivo, incluindo altos níveis de depressão.

Santos e Rodrigues (2020), destacam que a predominância de repercussões psicológicas negativos, muito se deram por causa da quarentena e do isolamento social, notou-se em seu estudo a frequência de caso de depressão.

Moni et al. (2021), mostram em sua pesquisa que o aumento do sofrimento psicológico, principalmente casos de depressão, está associado com as dificuldades financeiras. Isso pode ser explicado pelo surgimento de uma sensação de incerteza e falta de segurança durante quarentena e o isolamento social.

Richter et al. (2021), destacam diversas doenças mentais, entre elas a depressão, no início a sintomatologia se dar pela falta de vontade para realizar atividades que davam prazer, energia reduzida e cansaço constante.

Filho e Lima (2020), em sua pesquisa obtiveram resultados que apontam o isolamento social e a solidão como fatores para o aumento do risco de depressão e ansiedade. A pesquisa conclui que crianças e adolescentes podem experimentar depressão e ansiedade, no entanto os profissionais de saúde, principalmente aqueles que atuam na linha de frente, o impacto psicológico também é significativo, concluindo que ansiedade e depressão foram mais prevalentes entre a enfermagem do que entre os médicos.

Rodríguez (2020), afirmou em sua pesquisa que a crise causada pela pandemia COVID-19 impacta em vários níveis, no âmbito emocional por exemplo o distanciamento social e atividades de auto isolamento podem levar a e afetar problemas de saúde mental pré-existente, como a depressão. 
A depressão combinada com a ansiedade, se destacou no estudo de Nochaiwong et al. (2021), demonstrando a associação de quadros de desestabilidade emocional pós Covid-19.

\subsection{Impactos obtidos pela Covid -19.}

\subsubsection{Aumento dos casos de suicídio.}

Santos e Rodrigues (2020), destacam um dos dados mais preocupantes, no que diz respeito aos impactos desta pandemia, que são os relatos de suicídio. Segundo ele a pessoa que pensa em tirar sua vida, acredita que não existem soluções para os seus problemas e, normalmente, dá sinais de desequilíbrio emocional.

Richter et al. (2021), segundo a sua pesquisa as taxas de suicídio e as taxas de tentativas de suicídio em vários países não aumentaram em comparação com os tempos pré-pandêmicos, segundo o autor a taxa de suicídios não foi influenciada pandemia.

Filho e Lima (2020), associam o aumento da do consumo de drogas recreativas, com ênfase no álcool ao aumento dos casos de suicídios, já que segundo o mesmo o uso descontrolado de álcool é um fator de risco para tentativas de suicídio. Ainda no que diz respeito a temática associou o aumento da taxa de suicídios ao desemprego, essa condição pode acarreta em sérios danos à saúde mental e até mesmo ao suicido.

Rodríguez (2020), observou que a aplicação de certas medidas (como quarentena ou distanciamento Social) são essenciais na prevenção da doença, em contraponto enfatizou que essa medida tem desempenham um papel fundamental nos casos de suicídio. A teoria basicamente é a seguinte, pessoas que experimentam ideias suicidas na maioria das vezes priva-se da companhia de outras pessoas e à medida que aumenta o ato de se desconectar de outras pessoas por consequência aumenta $\mathrm{o}$ risco de suicídio, desta forma o pensamentos e comportamentos suicidas estão associados ao isolamento social e solidão, o que que leva a pensar que a pandemia contribuiu para que as pessoas ficassem isoladas e consequentemente esse cenário permite que as mesma experimentem de ideias suicidas.

Galea e Ettman (2021), afirmam que uma das razões pela qual a saúde mental é importante para a mortalidade em geral, principalmente em uma época de Covid-19 é a sua ligação causal direta entre transtornos de ansiedade e humor, que se manifesta como dano autoinfligido e morte por suicídio.

A doença por Covid-19 se estigma, o que tem levado pessoas infectadas a terem medo de enfrentar a patologia, ou seja, procurar atendimento, acabam por omitir o diagnóstico, o que tem aumentando a contaminação. PO outro lado, há aqueles que não procuram atendimento médico por medo de se infectar nesses ambientes. A experiência de perder um ou mais entes queridos também é um fator que tem afetado a vida de muitas pessoas. A junção dos fatores anteriormente citados tem contribuído para o aumento do suicídio entre idosos afetadas pela doença nos dois anos seguintes ao surto (Prado e Freitas, 2020).

\subsubsection{Aumentos do uso de drogas recreativas.}

Pavani et al. (2020), observou que houve um aumento considerável do uso de drogas recreativas, destacou o álcool e o tabaco.

Alvarez e Jorge (2021), destacou que a pandemia causou diversos impactos na sociedade, por exemplo, distúrbios comportamentais que colocam a vida em risco como uso de substâncias psicoativas, que inclui o consumo de álcool, tabaco e soníferos (medicamentos, suplementos, infusões), entre outros. Essas sustâncias agem no sistema nervoso central e causam alterações na função fisiológica cerebral. Essas alterações interferem temporariamente no humor, consciência, comportamento e percepção do indivíduo. 
Moni et al. (2021), em sua pesquisa associam o sofrimento psicológico, causado pelas incertezas da pandemia ao aumento do uso de álcool como um artefato, uma forma de lidar com os impactos da Covid-19, no entanto, em contraponto era provável que o aumento do uso de álcool piorasse o sofrimento psicológico.

Digno de grande ênfase, segundo Filho e Lima (2020), é o aumento do consumo de álcool durante a pandemia. Segundo pesquisa da companhia americana Nielsen, a venda de bebidas alcoólicas aumentou 55\% até o fim de março de 2020 quando comparado com o mesmo período no ano anterior.

Segundo o trabalho Pinheiro e Kocourek (2020), A Organização Mundial de Saúde, afirma que alguns transtornos mentais pré-existentes tendem ao agravamento após uma situação de catástrofe. E que além do agravamento desses transtornos, existem outras situações ligadas a pandemia, principalmente quando se associa ao luto, por exemplo o uso abusivo de álcool e outras drogas, embora cada pessoa enfrentem as situações de perdas afetivas de formas individuais e diferentes.

A frustração exacerbada não estava conectada à rotina normal da população e isso pode levar o indivíduo a desenvolver um desejo maior por bebidas álcool ou consumir substâncias psicoativas de forma abusiva. Diante de tal cenário é nítido que sintomas, como: desesperança, irritabilidade, alterações no apetite e distúrbios do sono, sejam observados em pessoas que lidam com a dor do luto e os impactos da pandemia como uso abusivo de drogas.

\subsubsection{Aumento dos transtornos de estresse pós-traumático.}

Pavani et al. (2020) em seu estudo destacou o aumento dos transtornos de estresse pós-traumático como consequência da pandemia, sintomas como automutilação, irritabilidade, medo e são observados com mais frequência na população.

Alvarez e Jorge (2021), destacam que as pessoas geralmente apresentam sintomas de estresse pós-traumático, que inclui sentir-se estressado, apresentando sintomas de desconforto Peri traumático leve, moderado ou grave, fadiga e altos níveis de excitação.

Santos e Rodrigues (2020), em sua pesquisa, estacou-se o transtorno de estresse pós-traumático, afirmou que esse transtorno pode acontecer após o término do período da quarentena, está correlacionado com os danos e perdas obtidos durante a pandemia como quando ocorre o óbito de pessoas da família ou problemas financeiros ocasionado pela perda da renda.

Moni et al. (2021), afirmam que aqueles infectados com Covid-19 apresentaram níveis mais elevados de sofrimento psíquico e sintomas de estresse pós-traumático quando em comparação com aqueles não infectados.

De acordo com Nochaiwong et al. (2021), há uma prevalência combinada de problemas de saúde mental durante a pandemia de Covid-19, e esta ainda é maior do que antes do surto.

De modo geral, pode - se observar que as ocorrências de ordem emocionais por vezes não são advindas diretamente da doença em si, dos sintomas e sinais, mas estão fortemente associadas a todas as desordens biológicas, psicológicas e sociais que a pandemia acabou trazendo nos últimos dois anos, tal achado se confirma no estudo realizado por Varma et al. (2021), que afirma que fatores biológicos e sociais, repercutiram fortemente sobre a saúde emocional no período da pandemia, e que os grupos que mais tem risco de desenvolver problemas emocionais, são aquele que já tinham a pré - existência de tais fatores.

\section{Considerações Finais}

A pandemia de Covid-19, impactou o mundo inteiro, não somente no que se refere aos problemas de saúde em si, as sequelas vão desde a perda de milhares de pessoas no mundo inteiro em decorrência dos desfechos clínicos da patologia, mas também através da saúde mental, pode -se considerar que o mundo adoeceu.

Este estudo teve como objetivo analisar o impacto na saúde mental da população em função da Covid-19; dessa forma foi possível observar o aumento de queixas como: ansiedade, depressão, estresse pós traumático. Além das já citadas, ainda foi observada o aumento do abuso de drogas, assim como a propensão ao suicídio, e o ato em si. 
Diante disso, se faz necessário que o sistema de saúde construa políticas públicas que venham a atender a população na saúde mental pós Covid-19, além da divulgação de informações corretas sobre o tratamento e a prevenção à patologia, uma vez que a melhor forma de prevenção é a educação em saúde.

Não se teve a pretensão de esgotar o tema proposto, mas buscou - se trazer uma reflexão a respeito da saúde mental em tempos de pandemia, além de trazer fomento para a comunidade academia para futuros estudos. Dessa forma sugere -se novas pesquisas que venham a avaliar as ações de saúde voltadas para a saúde mental da população, afim de se desenvolver estratégias para a melhora do cenário atual.

\section{Referências}

Alvarez, C. A., \& Jorge, I. T. (2021). Impacto del COVID-19 en la salud mental: revisión de la literatura. Repertório de Medicina y Cirugíia. 2021; 30(1) 2129 10.31260/RepertMedCir.01217372.1180.

Azevedo, D. K. L., Silva, C. M. P., \& Maia, A. L. (2021). O papel da gestão de enfermagem na implementação da meta de cirurgia segura: uma revisão de literatura. Research, Society and Development, 10(14), e584101422711, http://dx.doi.org/10.33448/rsd-v 10i14.22711.

Brooks, S. K., Webster, R. K., Smith, L. E., Woodland, L., Wessely, S., Greenberg, N., \& Rubin, G. J. (2020). O impacto psicológico da quarentena e como reduzi-lo: revisão rápida das evidências. Alumni Medicina, Centro Hospitalar Universitário do Algarve, Unidade de Faro https://doi.org/10.1016/S01406736(20)30460-8.

Filho, A. D. S., \& Lima, A. (2020). Saúde mental na pandemia covid 19. Subsecretaria de Saúde Gerência de Informações Estratégicas em Saúde CONECTASUS. Secretaria de estado da Saúde de Goiás. https://www.saude.go.gov.br >produtos-técnicos.

Galea, S., \& Ettman, C. K. (2021). Mental Health and Mortality in a Time of COVID-19. American Journal of Public Health . Opinions, Ideas, \& Practice. Supplement 2, 2021, 111(2). https://ajph.aphapublications.org/doi/pdfplus/10.2105/AJPH.2021.306278.

Gerhardt, T. E., \& Silveira, D. T. (2009). Métodos de pesquisa. Planejamento e gestão para o desenvolvimento rural da SEAD/UFRGS. Universidade Federal do Rio Grande do Sul. https://lume.ufrgs.br/bitstream/handle/10183/52806/000728684.pdf?sequence=1\&isAllowed=y.

Hallal, P.C., Horta, B. 1., Barros, A. J. D., Dellagostin, O. A., Hartwig, F. P., Pellanda, L. C., Struchiner, C. J., Burattini, M. N., Silveira, M. F., Menezes, A. M. B., Barros, F. C., \& Victora, E. C. G. (2020). Evolução da prevalência de infecção por COVID-19 no Rio Grande do Sul, Brasil: inquéritos sorológicos seriados. Ciência \& Saúde Coletiva, 25(Supl.1):23952401, 2020. https://doi.org/10.1590/1413-81232020256.1.09632020.

Meaklim, H., Junge, M., \& Jackson, M. L. (2021). Poor Sleep Quality and Its Relationship with Individual Characteristics, Personal Experiences and Mental Health during the COVID-19 Pandemic. International Journal of Environmental Research and Public Health. 6030. https://doi.org/10.3390/ij erph18116030.

Moni, A. S. B., Abdullah, S., Abdullah, M. F. I. L. B., Kabir, M. S., Sheikh, M. A., Sultana, F., Salehin, M., Islam, S. M. S., Cross, W., \& Rahman, M. A. (2021). Sofrimento psicológico, medo e enfrentamento entre os malaios durante a pandemia Covid-19. PLoS ONE 16 (9): e0257304. https://doi.org/ 10.1371/journal.pone.0257304.

Nochaiwong, S., Ruengorn, C., Thavorn, K., Hutton, B., Awiphan, R., Phosuya, C., Ruanta, Y., Wongpakaran, Nahathai., \& Wongpakaran, T. (2021). Global prevalence of mental health issues among the general population during the coronavirus disease-2019 pandemic: a systematic review and meta-analysis. Scientifc Reports. (2021) 11:10173. https://doi.org/10.1038/s41598-021-89700-8.

Ouyang, H., Wen, J., Gu, W., Shen, H., \& Song, K. Evaluation of the effect of the Covid-19 pandemic on clinical characteristics and psychological status in internet consultation respondentes. Science Progress 2021, 104(2) 1-16. https://doi.org/10.1177/00368504211014696.

Pavani, F. M., Silva, A. B., Olschowsky, A., Wetzel, C., Nunes, C. K., \& Souza, L. B. (2021). Covid-19 e as repercussões na saúde mental: estudo de revisão narrativa de literatura. Revista Gaúcha de Enfermagem. 2021;42(esp):e20200188. https://doi.org/10.1590/1983- 1447.2021.20200188.

Pinheiro, G. E. W., \& Kocourek, S. (2020). Saúde mental em tempos de pandemia: qual o impacto do Covid-19? Revista Cuidarte. 2020;11(3):e1250. http://dx.doi.org/10.15649/cuidarte.1250.

Prado, A. S., \& Freitas, J. L. (2020). Social distancing during pandemics: Suicide risk and prevention in the face of psychosocial impacts of COVID-19. Estudos de Psicologia, 25(2), 157-166. 10.22491/1678-4669.20200016.

Raony, I., Saggioro, C. F., Pandolfo, P., Araujo, E. G., Bomfim, P. O. S., \& Savino, W. (2020). Interações psico-neuroendócrino-imunes em COVID-19: impactos potenciais na saúde mental. Frontiers In Immunology. https://doi.org/10.3389/fimmu.2020.01170.

Richter, D., Riedel, H. S., \& Zürcher, S. J. (2021). Mental health problems in the general population during and after the first lockdown phase due to the SARS-Cov-2 pandemic: rapid review of multi-wave studies. Epidemiology and Psychiatric Sciences. 30(7), 1-9. https://doi.org/10.1017/ S2045796021000160

Rodríguez, J. H. (2020). Impacto de la Covid-19 sobre la salud mental de las personas. Medicentro Electrónica 24(3) http://scielo.sld.cu/scielo.php?script=sci_arttext\&p id=S1029-30432020000300578.

Santos, M. F., \& Rodrigues, J. F. S. (2020). COVID-19 e repercussões psicológicas durante a quarentena e o isolamento social: uma revisão integrativa. Revista Nursing (Säo Paulo) ; 23(265): 4095-4100 https://doi.org/10.36489/nursing.2020v23i265p4095-4100. 
Research, Society and Development, v. 10, n. 16, e260101623988, 2021

(CC BY 4.0) | ISSN 2525-3409 | DOI: http://dx.doi.org/10.33448/rsd-v10i16.23988

Souza, M. T., Silva, M. D., Carvalho, R. (2010). Revisão integrativa: o que é e como fazer. Einstein. 2010; 8(1):102-6. https://www.scielo.br/j/eins/a/ZQTBkVJZqcWrTT34cXLjtBx/?format=pdf\&lang=pt.

Varma, P., Burge, M., Meaklim, H., Junge, M., \& Jackson, M. L. (2021). Poor Sleep Quality and Its Relationship with Individual Characteristics, Personal Experiences and Mental Health during the COVID-19 Pandemic. International Journal of Environmental Research and Public Health. 2021, 18, 6030. https://doi.org/10.3390/ijerph18116030. 